\title{
Effects of oral phosphatidic acid feeding with or without whey protein on muscle protein synthesis and anabolic signaling in rodent skeletal muscle
}

C. Brooks Mobley ${ }^{1}$, Troy A. Hornberger², Carlton D. Fox', James C. Healy ${ }^{1}$, Brian S. Ferguson ${ }^{1}$, Ryan P. Lowery ${ }^{3}$, Rachel M. McNally ${ }^{2}$, Christopher M. Lockwood ${ }^{4}$, Jeffrey R. Stout ${ }^{5}$, Andreas N. Kavazis', Jacob M. Wilson ${ }^{3}$

and Michael D. Roberts ${ }^{*}$

\begin{abstract}
Background: Phosphatidic acid (PA) is a diacyl-glycerophospholipid that acts as a signaling molecule in numerous cellular processes. Recently, PA has been proposed to stimulate skeletal muscle protein accretion, but mechanistic studies are lacking. Furthermore, it is unknown whether co-ingesting PA with other leucine-containing ingredients can enhance intramuscular anabolic signaling mechanisms. Thus, the purpose of this study was to examine if oral PA feeding acutely increases anabolic signaling markers and muscle protein synthesis (MPS) in gastrocnemius with and without whey protein concentrate (WPC).
\end{abstract}

Methods: Overnight fasted male Wistar rats ( 250 g) were randomly assigned to four groups: control (CON, $n=6-13)$, PA (29 mg; $n=8)$, WPC (197 mg; $n=8)$, or PA + WPC $(n=8)$. Three hours post-feeding, gastrocnemius muscle was removed for markers of Akt-mTOR signaling, gene expression patterns related to skeletal muscle mass regulation and metabolism, and MPS analysis via the SUnSET method.

Results: Compared to CON rats, PA, WPC and PA + WPC resulted in a significant elevation in the phosphorylation of mTOR (Ser2481) and rps6 (Ser235/236) $(p<0.05)$ in the gastrocnemius though there were no differences between the supplemented groups. MPS levels in the gastrocnemius were significantly $(p<0.05)$ elevated in WPC versus CON rats, and tended to be elevated in PA versus CON rats $(p=0.08)$, though MPS was less in PA + WPC versus WPC rats $(p<0.05)$ in spite of robust increases in mTOR pathway activity markers in the former group. $C_{2} C_{12}$ myoblast data agreed with the in vivo data herein showing that PA increased MPS levels $51 \%(p<0.001)$ phosphorylated p70s6k (Thr389) levels $67 \%$ $(p<0.001)$.

Conclusions: Our results are the first in vivo evidence to demonstrate that PA tends to increases MPS $3 \mathrm{~h}$ post-feeding, though PA may delay WPC-mediated MPS kinetics within a $3 \mathrm{~h}$ post-feeding window.

\footnotetext{
* Correspondence: mdr0024@auburnedu

'School of Kinesiology, Auburn University, Auburn, AL, USA

Full list of author information is available at the end of the article
}

(c) 2015 Mobley et al. Open Access This article is distributed under the terms of the Creative Commons Attribution 4.0 International License (http://creativecommons.org/licenses/by/4.0/, which permits unrestricted use, distribution, and reproduction in any medium, provided you give appropriate credit to the original author(s) and the source, provide a link to the Creative Commons license, and indicate if changes were made. The Creative Commons Public Domain Dedication waiver (http://creativecommons.org/publicdomain/zero/1.0/) applies to the data made available in this article, unless otherwise stated. 


\section{Background}

Skeletal muscle growth is controlled by several intricate processes dictated in large part by nutrient and intramuscular mechano-responsive factors [1]. To this end, the mammalian target of rapamycin (mTOR) is thought to be a nodal point for controlling skeletal muscle hypertrophy. While mTOR activation is complex, a simplistic overview of this process is as follows [2]: a) mTOR complex 1 (mTORC1), which is comprised of mTORRaptor- mLST8, can be activated by muscle contraction and nutritional factors; b) activated mTORC1 then acts to phosphorylate and activate p70s6 kinase (p70s6k) while hyper-phosphorylating eukaryotic initiation factor 4E (eIF4E)-binding proteins (4EBP-1/2); c) activated p70s6k phosphorylates and activates ribosomal protein s6 (rps6), while hyperphosphorylated 4EBP1/2 become inactive thus facilitating ribosomal assembly; and d) activated rps6 further increases ribosomal assembly via enhanced 5'-cap-dependent messenger RNA (mRNA) translation of selective genes. These aforementioned processes ultimately result in an elevation in skeletal muscle protein synthesis (MPS) and, if stimulated repetitively with resistance exercise and ample nutrition, lead to an increase in skeletal muscle accretion.

Phosphatidic acid (PA) is a diacyl-glycerophospholipid that is enriched in eukaryotic cell membranes and it can act as a signaling lipid [3]. For instance, it has been shown that PA regulates a wide array of cellular processes including but not limited to proliferation, differentiation, survival signaling, cytoskeletal rearrangement and vesicular trafficking (reviewed in [4]). PA can also elicit anabolic responses in cells. In this regard, a hallmark mechanistic study performed by Fang et al. [5] demonstrated that PA activates mTOR by binding to the FKBP12-rapamycin binding (FRB) domain on mTOR. Follow-up studies by Hornberger et al. [6] revealed that mechanical stretching of skeletal muscle promotes an increase in intramuscular PA levels and this effect was associated with the activation of mTOR signaling (e.g. increased p70s6k phosphorylation), and that eccentric contraction increases skeletal muscle PA levels which, in turn, activate mTOR signaling [7]. More recent data from Hornberger's group suggests that the $\zeta$ isoform of diacylglycerol kinase, which synthesizes intramuscular PA via the phosphorylation of diacylglycerol, is largely responsible for the increase in PA levels and the activation of mTOR signaling that occurs in response to stretch [8]. However, others have suggested that PA does not directly bind to mTOR, but rather activates the MAP kinase pathway proteins Erk1/2 via its conversion into lysophosphatidic acid (LPA) which ultimately results in mTOR activation [9]. Likewise, others have shown that PA binds to p70s6k to exhibit its biological effects in an mTOR-independent fashion [10]. Notwithstanding, and in spite of these divergent findings, there is ample in vitro evidence to suggest that PA increases mTORC1 signaling.

Given the ability of biosynthesized PA to activate the intramuscular mTORC1 signaling, there is intense interest for the potential of PA supplementation to act as an ergogenic/muscle-building aid. To this end, two recent human studies supplemented participants with $750 \mathrm{mg}$ of soy-derived PA over an 8-week resistance training period. Hoffman et al. [11] reported that PA supplementation increased whole-body lean body mass (LBM) by $1.7 \mathrm{~kg}$, whereas the placebo group demonstrated no relative change in LBM ( $0.1 \mathrm{~kg} ; p=0.065$ between groups). Joy et al. [12] performed a similar 8-week study with more participants and supervised training sessions, and reported that soy-derived PA supplementation significantly increased LBM by $2.4 \mathrm{~kg}$, whereas the placebo group demonstrated marginal increases in LBM $(1.2 \mathrm{~kg}$; $p<0.05$ between groups). Joy et al. also used an in vitro approach to demonstrate that stimulating differentiated myoblasts with soy-derived PA elicited over a 6-fold increase in p70s6k (Thr389) phosphorylation which is indicative of mTOR activation. While both studies suggest that soy-derived PA supplementation is effective at augmenting resistance exercise-induced skeletal muscle hypertrophy, neither study measured the effects of PA on MPS levels and/or mTOR signaling events in vivo. Likewise, it remains to be determined if other mTORmodulating ingredients (i.e., intact dietary protein sources rich in L-leucine) provide a synergistic effect with regards to activating mTOR and downstream events related to increasing MPS. Leucine activates mTOR through RAG GTPases which, in the presence of leucine, bind to Raptor and increase mTORC1 complex localization to vesicles to increase its kinase activity [13]. PA is thought to independently activate mTOR through competitive binding with the mTOR inhibitor FKBP38; both which bind to the FRB domain [14]. Thus, given that these are two modes whereby mTORC1 kinase activity can be independently activated, it stands to reason that whey protein could synergistically activate mTOR if co-ingested with PA.

Therefore, the purpose of this study was to examine if PA acutely increases anabolic signaling markers and muscle protein synthesis (MPS) in gastrocnemius with and without whey protein concentrate (WPC) supplementation. As a side, we also sought to examine the skeletal muscle mRNA response to PA and/or WPC ingestion in an exploratory manner given that no information to our knowledge exists on how PA ingestion affects the skeletal muscle transcriptomic response. To this end, and independent of mTOR signaling and MPS, we examined key genes involved in muscle mass maintenance [myostatin (Mstn) and p21Cip1], metabolism 
(PGC-1 $\alpha$ and GLUT-4), and skeletal muscle atrophy (Atrogin-1 and MuRF-1) in an exploratory manner to examine if PA with or without whey protein affected these markers.

\section{Methods}

Animals and feeding protocols

All experimental procedures described herein were approved by Auburn University's Institutional Animal Care and Use Committee (protocol\# 2013-2378). Male Wistar rats ( 250 g) were purchased from Harlan Laboratories and were allowed to acclimate in the animal quarters for 5 days prior to experimentation. Briefly, animal quarters were maintained on a $12 \mathrm{~h}$ light: $12 \mathrm{~h}$ dark cycle, at ambient room temperature, and water and standard rodent chow (18.6 \% protein, $44.2 \%$ carbohydrate, $6.2 \%$ fat; Teklad Global \#2018 Diet, Harlan Laboratories, Indianapolis, IN, USA) was provided to animals ad libitum.

The day prior to the acute feeding experiments, food was removed from home cages resulting in an $18 \mathrm{~h}$ overnight fast. The morning of experimentation, animals were removed from their quarters between 0800-0900, transported to the Molecular and Applied Sciences Laboratory and were allowed to acclimate for approximately $3-5 \mathrm{~h}$. Thereafter, rats were gavage-fed either:

1) $\mathrm{CON}: 1 \mathrm{ml}$ of tap water

2) PA: 0.029 g soy-derived PA (S-PA, Mediator ${ }^{\circ}$, Chemi Nutra, Austin, TX, USA) suspended in $1 \mathrm{ml}$ of tap water; this being a human equivalent dose of $1.5 \mathrm{~g}$ per the species conversion calculations of ReaganShaw et al. [15]

3) WPC: $0.193 \mathrm{~g}$ WPC (standardized to $80 \%$, donated graciously by C.M.L.) suspended in $1 \mathrm{ml}$ of tap water; this being a human equivalent dose of $10 \mathrm{~g}$

4) PA + WPC: 0.029 g soy-derived PA + $0.193 \mathrm{~g}$ WPC suspended in $1 \mathrm{ml}$ of tap water

Of note, select data from group \#3 above (WPC) published as control group in a previously published study by our group [16], but was used as a reference to examine how PA and PA + WPC affected post-feeding markers of muscle anabolism relative to WPC alone. It should also be noted that, while a $10 \mathrm{~g}$ human equivalent dose may be considered too low with regards to being able to increase skeletal muscle MPS given that $10 \mathrm{~g}$ of WPC delivers $\sim 1 \mathrm{~g}$ of leucine to humans, we have consistently observed this dose of whey protein to increase MPS in rat skeletal muscle in pilot experiments (Additional file 1: Figure S1 in Mobley et al. [16]) as well as previously published data [16].

The gavage feeding procedure involved placing the animals under light isoflurane anesthesia for approximately $1 \mathrm{~min}$ while gavage feeding occurred. Following gavage feeding, rats were allowed to recover $180 \mathrm{~min}$ prior to being euthanized under $\mathrm{CO}_{2}$ gas. Of note, animals were injected (i.p.) with puromycin dihydrochloride $30 \mathrm{~min}$ prior to euthanasia $(5.44 \mathrm{mg}$ in $1 \mathrm{ml}$ of diluted in phosphate buffered saline; Ameresco, Solon, OH, USA) to determine skeletal MPS levels via the SUnSET method [17].

Immediately following euthanasia, approximately two $50 \mathrm{mg}$ pieces of mixed gastrocnemius muscle were harvested using standard dissection techniques and placed in RIPA buffer [Tris base; $\mathrm{pH} 8.0, \mathrm{NaCl}, \mathrm{NP}-40$, sodium deoxycholate, SDS with added $1 \mathrm{x}$ protease (Ameresco) and $1 \mathrm{x}$ phosphatase II and III inhibitors (G BioSciences, St. Louis, MO, USA)] and Ribozol (Ameresco) for immunoblotting and mRNA analyses, respectively. Gastrocnemius samples placed in radioimmunoprecipitation assay (RIPA) buffer were homogenized immediately upon extraction using a micropestle, insoluble proteins were removed with centrifugation at $500 \mathrm{xg}$ for $5 \mathrm{~min}$, and supernatants were assayed for total protein content using a BCA Protein Assay Kit (Thermo Scientific, Waltham, MA, USA) prior to immunoblotting sample preparation. Gastrocnemius samples placed in Ribozol were subjected to total RNA isolation according to manufacturer's instructions, and RNA concentrations were measured with a NanoDrop Lite (Thermo Scientific) prior to cDNA synthesis for mRNA analyses. Extra gastrocnemius muscle not processed during dissections was flash-frozen in liquid nitrogen and stored at $-80{ }^{\circ} \mathrm{C}$ for later potential analyses.

\section{Directed Akt-mTOR phosphoproteomics}

The PathScan ${ }^{\circ}$ Akt Signaling Antibody Array Kit (Chemiluminescent Readout; Cell Signaling, Danvers, MA, USA) containing glass slides spotted with antibodies was utilized to detect phosphorylated proteins predominantly belonging to the Akt-mTOR signaling network.

Briefly, gastrocnemius homogenates were diluted to $0.5 \mu \mathrm{g} / \mu \mathrm{l}$ using cell lysis buffer provided by the kit and assayed according to manufacturer's instructions. Slides were developed using an enhanced chemiluminescent reagent provided by the kit at 1 min exposure times for all chips, and spot densitometry was performed through the use of a UVP Imager and associated densitometry software (UVP, LLC, Upland, CA, USA). The calculation of each phosphorylated target was as follows:

(Density value of the target - density of negative control) / between-sample normalizing factor (which was the summation of all density values on the Chip for a given sample).

It should be noted that this high throughput antibody chip array for muscle phosphorylation markers was used rather than single antibodies due to resource constraints. Moreover, others in the literature have used 
chemiluminescent-based phosphoarray chips for screening purposes as we have utilized them herein $[18,19]$. Finally, prior publications from our laboratory using this method has shown that different doses of whey protein feedings in rats increases p-rps6, p-mTOR, and p-p70sk6 (Additional file 1: Figure S1 in Mobley et al. [16]), and these arrays are sensitive at detecting differences in phospho-signaling events that occur in rodents in response to cardiac ischemia-reperfusion [20].

\section{Western blotting}

As mentioned prior, the SUnSET method was employed in order to examine if different dietary blends differentially affected MPS. Briefly, gastrocnemius RIPA homogenates were prepared for Western blotting using $4 \mathrm{x}$ Laemmli buffer at a concentration of $2 \mu \mathrm{g} / \mu \mathrm{l}$. Thereafter, $20 \mu \mathrm{l}$ of prepped samples were loaded onto pre-casted $12 \%$ SDS-polyacrylamide gels (Bio-Rad, Hercules, CA, USA) and subjected to electrophoresis (60 min @ $200 \mathrm{~V})$. Proteins were then transferred (120 min @ 200 mAmps) to polyvinylidene difluoride membranes, and membranes were blocked for $1 \mathrm{~h}$ at room temperature with $5 \%$ nonfat milk powder in Tris-buffered saline + Tween-20 (TBST). For muscle samples, mouse anti-puromycin IgG (clone 12D10, 1:5,000; Millipore-Merck KGaA, Darmstadt, Germany) was incubated with membranes overnight at $4{ }^{\circ} \mathrm{C}$ in $5 \%$ bovine serum albumin (BSA) in TBST, and the following day membranes were incubated with antimouse IgG secondary antibodies (1:2,000, Cell Signaling, Danvers, MA, USA) at room temperature for $1 \mathrm{~h}$ prior to membrane development described below. Thereafter, membranes were stripped of antibodies via commercial stripping buffer (Restore Western Blot Stripping Buffer, Thermo Scientific), membranes were then re-blocked as described above and incubated with rabbit anti-betaactin (1:5,000; GeneTex, Inc., Irvine, CA, USA) as a normalizer protein overnight at $4{ }^{\circ} \mathrm{C}$ in $5 \%$ BSA in TBST, and the following day membranes were incubated with anti-rabbit IgG secondary antibodies (1:2,000; Cell Signaling) at room temperature for $1 \mathrm{~h}$ prior to membrane development.

Membrane development was performed using an enhanced chemiluminescent reagent (Amersham, Pittsburgh, PA, USA), and band densitometry was performed through the use of a gel documentation system and associated densitometry software (UVP, Upland, CA, USA).

\section{Real-time RT-PCR}

Gastrocnemius RNA was obtained using Ribozol (Ameresco, Solon, OH, USA) per the manufacturer's recommendations. Total RNA concentrations as well as RNA purity $(260 / 280$ OD ratio $>1.8)$ were analyzed using a Nanodrop Lite spectrophotometer (Thermo Scientific, Waltham, MA, USA), and $1 \mu \mathrm{g}$ of cDNA was synthesized using a commercial qScript ${ }^{\mathrm{Tm}} \mathrm{cDNA}$ SuperMix (Quanta Biosciences, Gaithersburg, MD, USA) per the manufacturer's recommendations. Following cDNA synthesis the resultant cDNA was diluted to $5 \mathrm{ng} / \mu \mathrm{l}$ using RNase-free water. Real-time PCR was performed using SYBR-green-based methods with genespecific primers designed using primer designer software (Primer3Plus, Cambridge, MA, USA). The forward and reverse primer sequences are as follows: [Myostatin (Mstn): forward primer 5' - ACGCTACCACGGAAACAATC -3', reverse primer 5' - CCGTCTTTCATGGGTTTGAT -3'; p21Cip1: forward primer 5' - AGCAAAGTATGCCGTC GTCT -3', reverse primer 5' - ACACGCTCCCAGACGT AGTT -3'; Atrogin-1: forward primer 5'-CTACGATGT TGCAGCCAAGA $-3^{\prime}$, reverse primer $5^{\prime}$ - GGCAGTCGA GAAGTCCAGTC - $3^{\prime}$; Muscle RING finger protein1(MuRF-1): forward primer 5' - AGTCGCAGTTTCGAA GCAAT-3', reverse primer 5' - AACGACCTCCAGACA TGGAC-3'; Glucose transporter-4 (Glut-4): forward primer 5'-GCTTCTGTTGCCCTTCTGTC -3 ', reverse primer 5' - TGGACGCTCTCTTTCCAACT -3'; Peroxisome proliferator-activated receptor gamma coactivator 1-alpha (Pgc-1 $\alpha$ ): forward primer 5'- ATGTGTCGCCTTCTTGC TCT- 3', reverse primer 5' - ATCTACTGCCTGGGGAC CTT -3'; $\beta 2$-microglobulin (B2M; housekeeping gene): forward primer 5' - CCCAAAGAGACAGTGGGTGT - 3', reverse primer 5'- CCCTACTCCCCTCAGTTTCC -3'] and SYBR green chemistry (Quanta). Fold-change values from the water (fasting) treatment were performed using the Livak method (i.e., 2- $\Delta \Delta \mathrm{CT}$ assuming $100 \%$ primer binding efficiency), where $2-\Delta \mathrm{CT}=$ [housekeeping gene $\mathrm{CT}$ - gene of interest CT] and 2- $\Delta \Delta \mathrm{CT}$ (or foldchange) $=[2-\Delta \mathrm{CT}$ value $/ 2-\Delta \mathrm{CT}$ average of water (fasting) treatment]. Primer efficiency curves for all genes were generated and efficiencies ranged between $90 \%$ and $110 \%$.

\section{Cell culture}

$\mathrm{C}_{2} \mathrm{C}_{12}$ myoblasts (ATCC; Manassas, Virginia) were plated at approximately $40 \%$ confluence and grown for $24 \mathrm{~h}$ in $10 \%$ FBS High Glucose DMEM with antibiotics $(100 \mu \mathrm{g} / \mathrm{ml}$ streptomycin and $100 \mathrm{U} / \mathrm{ml}$ penicillin; Sigma). At $18 \mathrm{~h}$ prior to the experiment, the myoblasts were switched to serum free high glucose DMEM (no antibiotics) and were approximately $90 \%$ confluent at the time of the experiment. As detailed previously, the myoblasts were then stimulated with $30 \mu \mathrm{M}$ of soyderived PA (S-PA, Mediator ${ }^{\circ}$, Chemi Nutra, Austin, TX, USA) or PBS as a control condition [12]. For measurements of mTOR signaling, myoblasts were collected at $30 \mathrm{~min}$ after stimulation. For measurements of protein synthesis, $1 \mu \mathrm{M}$ puromycin was added to the media during the final $30 \mathrm{~min}$ of a $60 \mathrm{~min}$ stimulation period, as previously described [17]. In all cases, myoblasts 
were collected in an ice-cold lysis buffer consisting of (40 mM Tris, pH 7.5; 1 mM EDTA; 5 mM EGTA; $0.5 \%$ Triton X-100; $25 \mathrm{mM} \beta$-glycerophosphate; $25 \mathrm{mM} \mathrm{NaF}$; $1 \mathrm{mM} \mathrm{Na} \mathrm{VO}_{4} ; 10 \mu \mathrm{g} / \mathrm{mL}$ leupeptin; and $1 \mathrm{mM}$ PMSF) and then centrifuged at $500 \mathrm{xg}$ for $5 \mathrm{~min}$. The resulting supernatants were then subjected to Western blot analyses for puromycin, total p70s6k and phosphorylated p70s6k (Thr389) as detailed previously [12].

\section{Statistics}

All data are presented as means \pm standard error. For in vivo data, statistics were performed between treatments using an ANOVA with protected LSD post hoc comparisons when applicable in order to avoid an inflated type I error rate. For in vitro data, treatment comparisons were made using independent samples t-tests. All statistics were performed using IBM SPSS version 22.0 and significance was determined at $p<0.05$.

\section{Results}

\section{PA and PA + WPC increase $3 \mathrm{~h}$ post-feeding} phosphorylation of select mTOR pathway substrates

There was no difference in p-Akt (Ser473) between treatments $(p>0.05$; Fig. 1a). Compared to CON, phosphorylated p-mTOR (Ser2481), which is a marker of mTOR autophosphorylation and activation [21], was approximately 2 -fold higher in PA and PA + WPC rats $(p<0.05$; Fig. 1b), though there was no difference in this marker between the latter two groups. Compared to CON, PA and PA + WPC led to a paradoxical decrease in $\mathrm{p}-4 \mathrm{EBP} 1$ (Thr37/46) $(p<0.05$; Fig. 1c). Compared to CON, pp70s6k (Thr389) was $\sim 80 \%$ higher in PA + WPC rats $(p<0.05$; Fig. $1 \mathrm{~d})$, whereas other treatments did not statistically differ from CON. Compared to CON, prps6 (Ser235/236) was approximately 2.3-3.1-fold higher in PA and PA + WPC rats $(p<0.05$; Fig. 1e), though again there was no difference in this marker between the latter two groups.

\section{PA and/or PA + WPC minimally affect $3 \mathrm{~h}$ post-feeding phosphorylation of other substrates related to protein synthesis}

All treatments equally affected the phosphorylation status of AMPK- $\alpha$ (Thr172), Erk (Thr202/Tyr204), PDK1 (Ser241), and PRAS40 (Thr246) (Fig. 2). Interestingly, PA increased p-GSK-3 $\alpha$ (Ser21) $60 \%$ compared to CON $(p<0.05$; Fig. 2 d). As reported previously [22], WPC increased the phosphorylation of GSK-3 $\alpha / \beta$ (Fig. $2 d$ \& e), though PA + WPC interestingly exacerbated this response compared to WPC alone.

\section{Effects of PA and PA + WPC on $3 \mathrm{~h}$ post-feeding MPS levels}

Of note, WPC rats have been used in a prior study whereby we reported the significant two-fold increase in MPS relative to CON [22]. However, in the current study samples were re-run and normalized to Ponceau staining instead of beta-actin normalization given that mixed gastrocnemius fiber types may potentially yield varying normalization values. As expected, WPC robustly increased MPS levels compared to CON rats confirming our prior data. Compared to CON rats, MPS trended to be higher in PA-fed rats ( $p=0.08$; Fig. 3). Interestingly, however, PA + WPC-fed rats exhibited lower MPS levels compared to WPC-fed rats $(p<0.05$, Fig. 3$)$.

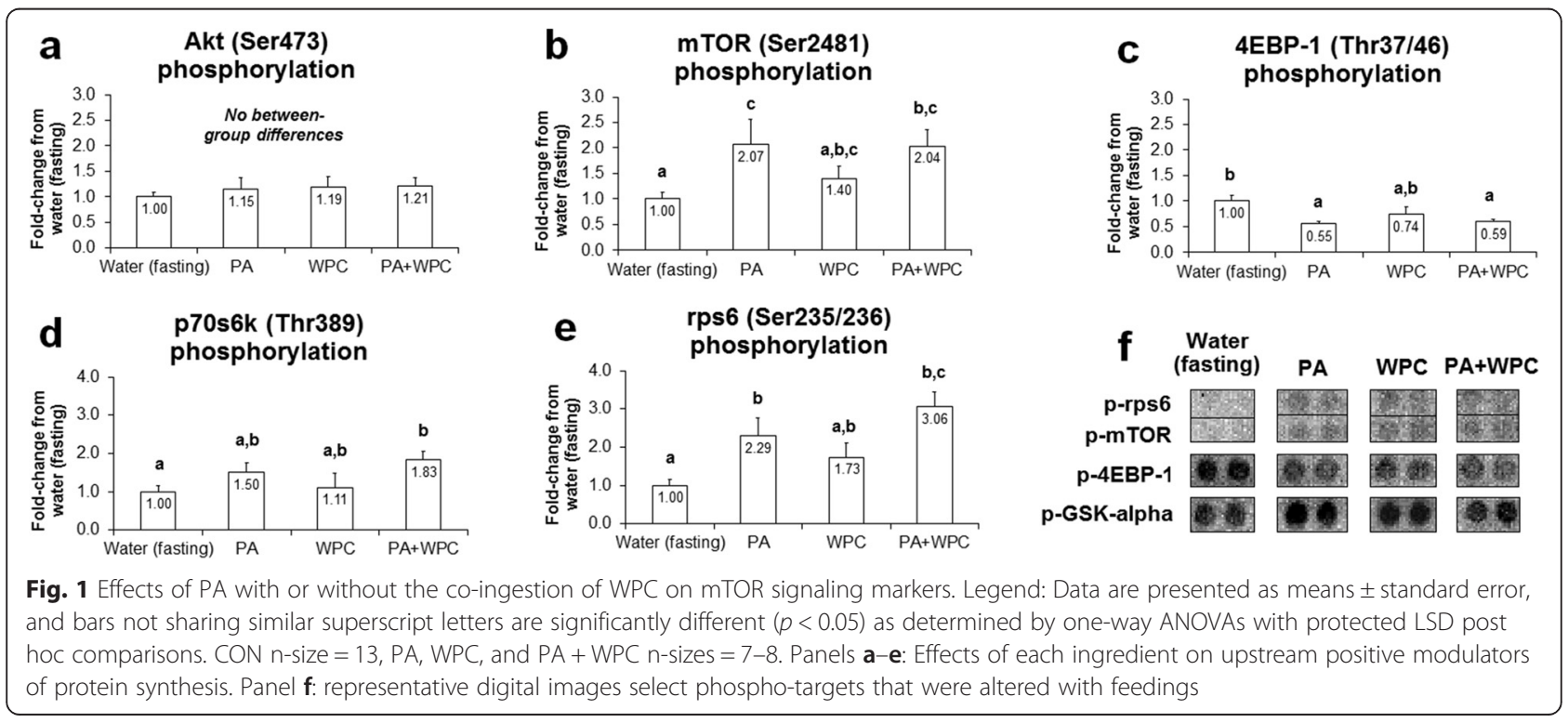




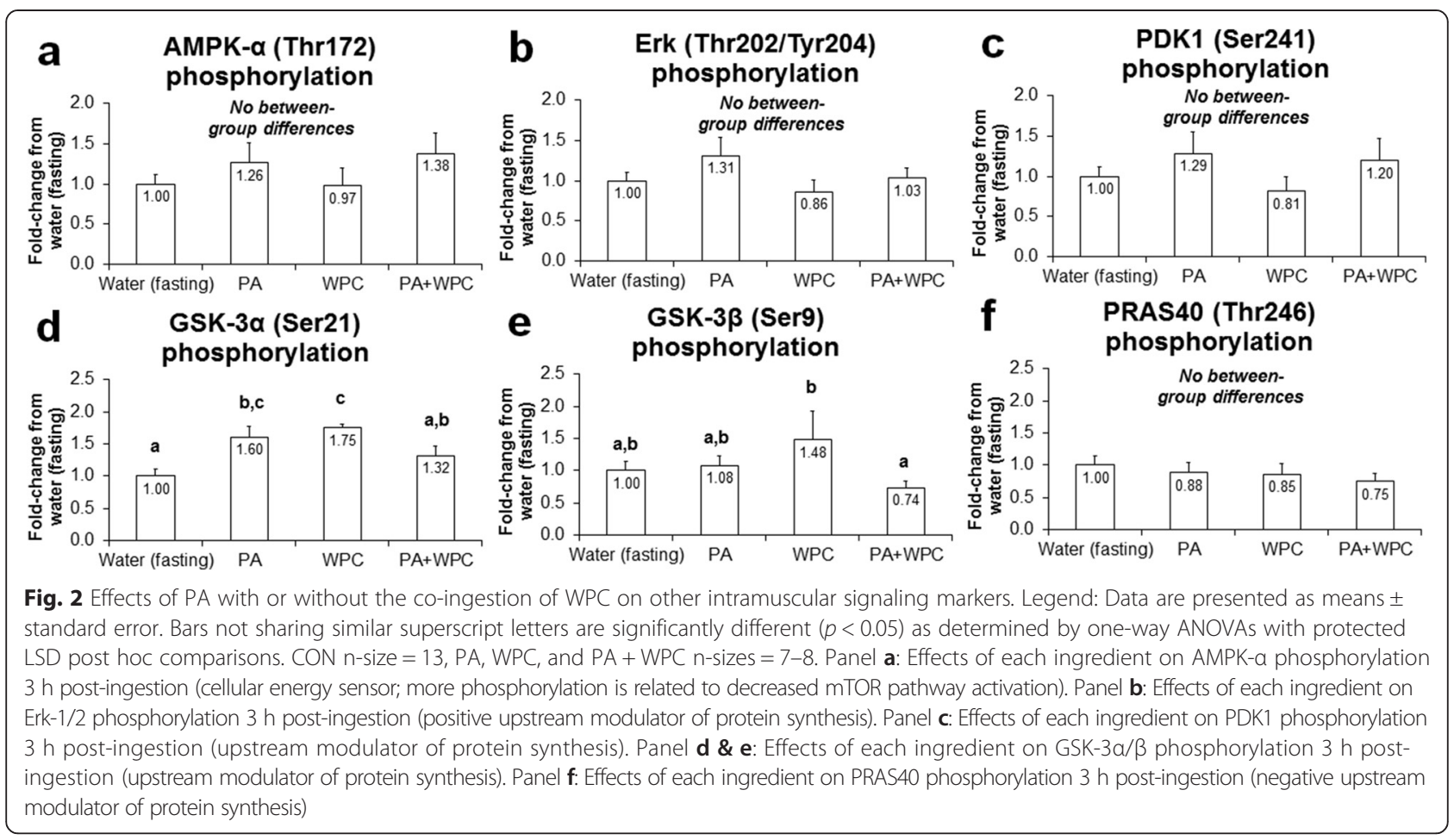

In vitro confirmation that PA increases MPS and mTOR signaling

p-p70s6k (Thr389) increased $67 \%$ in 30 min PA-treated myoblasts compared to the control condition $(p<0.001$;

Fig. 4a). Moreover, MPS levels were increased $51 \%$ in
60 min PA-treated myoblasts compared to the control condition $(p<0.001$; Fig. $4 \mathrm{~b})$. These data further validate the aforementioned in vivo results suggesting that the soy-derived PA studied herein increases mTOR signaling and likely increases MPS. a

\section{Muscle protein synthesis (SUnSET method)}

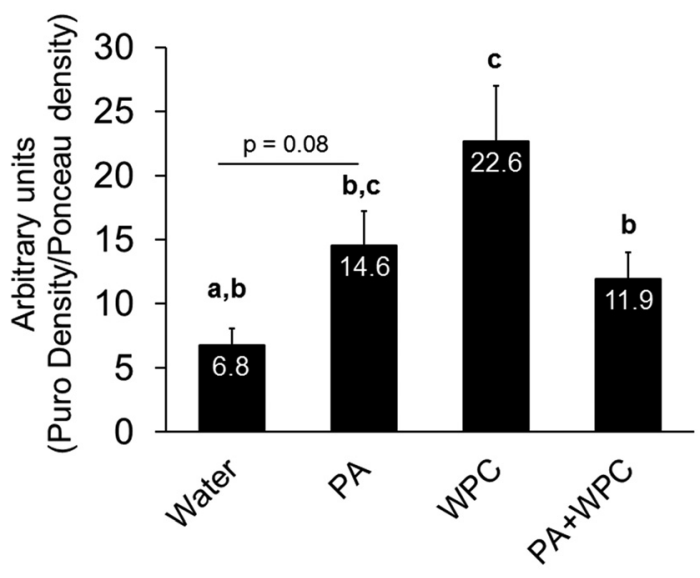

b

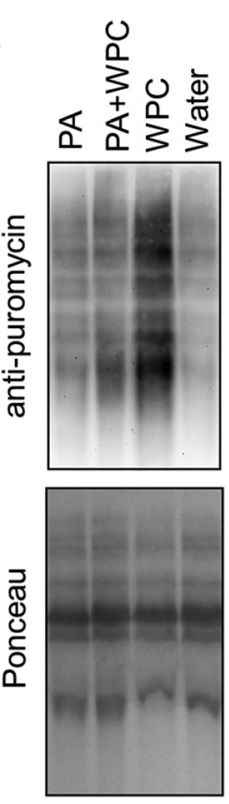

Fig. 3 Effects of PA with or without the co-ingestion of WPC on $3 \mathrm{~h}$ post-feeding MPS. Legend: Data are presented as means \pm standard error, and bars not sharing similar superscript letters are significantly different $(p<0.05)$ as determined by one-way ANOVAs with protected LSD post hoc comparisons. CON, PA, WPC, and PA + WPC n-sizes $=6-8$. Panel $\mathbf{a}$ : Effects of each treatment on $3 \mathrm{~h}$ post-feeding MPS levels. Panel $\mathbf{b}$ : Representative image of the SUnSET blot. PA, WPC, and PA + WPC all significantly increased MPS levels compared to fasting conditions 


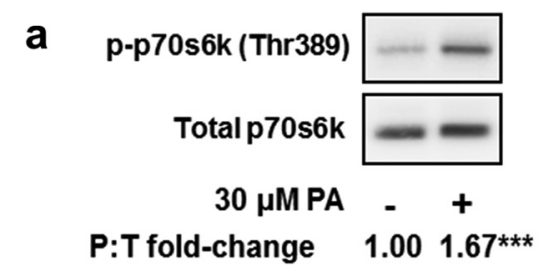

b

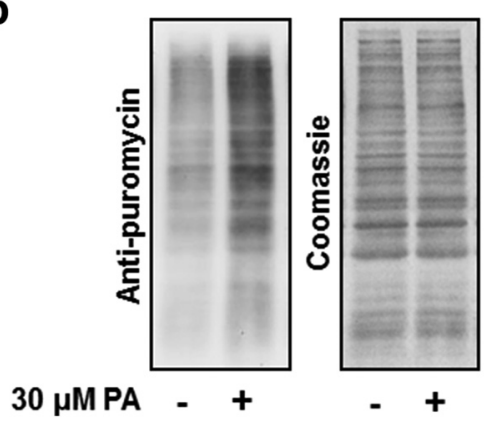

\section{Protein synthesis (SUnSET)}

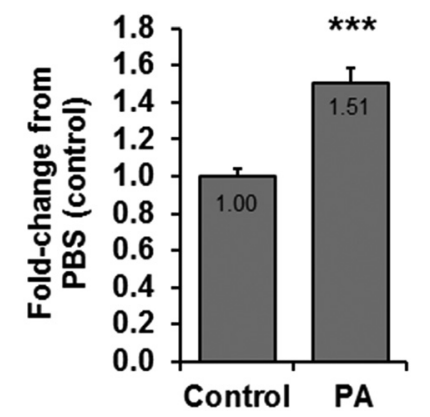

Fig. 4 PA increases $C_{2} C_{12}$ myoblast mTOR signaling and MPS. Legend: Data are presented as means \pm standard error. All data were obtained from 2-3 independent experiments ( $n=6-11$ wells per condition). Significant between-treatment differences ( $p<0.001)$ existed for $p$-p70s6k (Thr389) and MPS levels as determined by independent samples t-tests (denoted by different superscript letters). Panel a: $C_{2} C_{12}$ myoblasts were serum starved for $18 \mathrm{~h}$ and then stimulated for 30 min with $30 \mu \mathrm{M}$ PA, or PBS as a control condition. Measurements of mTOR signaling were assessed by evaluating the phospho to total ratio (P:T) for p-p70s6k (Thr389) and total p70s6k. Panel b: $\mathrm{C}_{2} \mathrm{C}_{12}$ myoblasts were treated as described in panel $\mathrm{a}$ and measurements of protein synthesis were performed by assessing puromycin incorporation during the final 30 min of a 60 min stimulation period. The Western blot membranes were stained with Coomassie Blue to verify equal loading of protein in all lanes

PA and PA + WPC increase select $3 \mathrm{~h}$ post-feeding mRNAs related to skeletal muscle anabolism and metabolism Beyond examining the effects that PA with or without WPC exhibited on mTOR signaling and MPS, we also were interested in prospectively exploring if these treatments acutely affected gene expression patterns related to skeletal muscle mass regulation and metabolism. Interestingly, PA or WPC did not cause a change in Mstn mRNA compared to CON, though PA + WPC rats presented statistically lower Mstn mRNA levels relative to the PA- and WPC-fed rats $(p<0.05$; Fig. $5 \mathrm{a})$; however, Mstn mRNA in WPC + PA did not change compared to the control group. Compared to CON, PA robustly increased p21Cip1 mRNA expression patterns over 5.6-fold ( $p<0.05$, Fig. 5b). Likewise, compared to CON, WPC and PA + WPC increased p21Cip1 mRNA expression over 3 -fold, though this only tended to be greater than fasting $(p<0.10)$ due to the high postfeeding variation in this transcript. No between-group differences existed in PGC- $1 \alpha$ mRNA levels (Fig. 5c). Compared to CON, PA increased Glut-4 mRNA levels $43 \%(p<0.05$; Fig. 5d) and PA + WPC increased Glut-4 mRNA expression patterns $\sim 60 \%(p<0.05$, Fig. $5 d)$. All treatments presented similar Atrogin-1 mRNA levels (Fig. 5e). Finally, PA + WPC increased MuRF-1 mRNA 2.3-fold relative to $\mathrm{CON}(p<0.05$, Fig. $5 f)$.

\section{Discussion}

This is the first study to demonstrate that PA, with or without WPC, increases the post-feeding phosphorylation of select skeletal muscle mTOR pathway substrates, and PA tends to increase MPS levels in vivo. Moreover, our in vitro data demonstrate that exogenous PA administration increases mTOR signaling and MPS in $\mathrm{C}_{2} \mathrm{C}_{12}$ myoblasts. These data complement the two human studies suggesting that 8 weeks of PA supplementation increases skeletal muscle mass [11, 12]. These findings are discussed in greater detail below.

\section{PA, with or without WPC, increases post-feeding mTOR pathway activity in vivo}

Our findings that PA, with or without WPC co-ingestion, increases post-feeding mTORC1 pathway activity and MPS in vivo supports other mechanistic literature examining the effects of PA on these variables. For instance, Fang et al. [5] treated HEK293 cells with PA and the authors demonstrated that: a) p70s6k activity increased; b) rapamycin (an mTOR inhibitor) blunted PA-induced increases in p70s6k activity; c) blunting serum-induced PA production in HEK293 cells with 1butanol reduced p70s6k activity; and d) PA has a high binding affinity for mTOR on the FRB rapamycinsensitive domain which, upon binding, increases the 

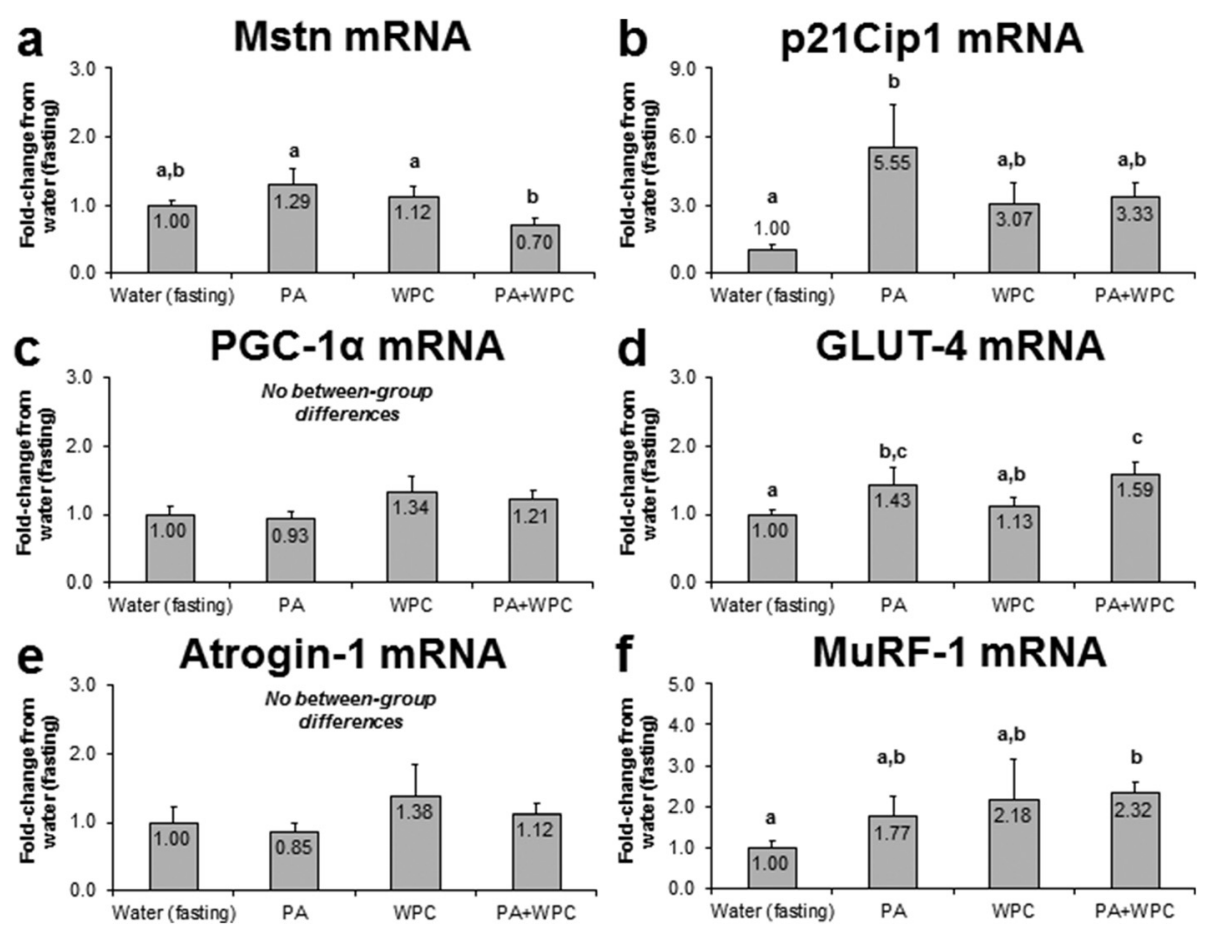

Fig. 5 Effects of PA with or without the co-ingestion of WPC on skeletal muscle mRNA expression patterns. Legend: Data are presented as means \pm standard error. Bars not sharing similar superscript letters are significantly different $(p<0.05)$ as determined by one-way ANOVAs with protected LSD post hoc comparisons. CON n-size $=13$, PA, WPC, and PA + WPC n-sizes $=7-8$. Panels a $\boldsymbol{\&} \mathbf{b}$ : Genes linked to skeletal muscle hypertrophy. Panels $\mathbf{c} \boldsymbol{\&} \mathbf{d}$ : Genes linked to skeletal muscle metabolism. Panels e \& f: Genes linked to skeletal muscle atrophy (Atrogin-1) and remodeling (MuRF-1)

phosphorylation of mTOR-dependent substrates such as p70s6k (Thr389). The aforementioned study also demonstrated that PA levels in HEK293 cells had no effect on p-Erk1/2 (Thr202/Tyr204) or p-Akt (Ser473) levels.

The effects of PA in rat skeletal muscle presented herein are in agreement with the aforementioned data. Specifically, we demonstrated that: a) PA or PA + WPC did not alter $3 \mathrm{~h}$ post-feeding p-Akt (Ser473) or pErk1/2 (Thr202/Tyr204) levels relative to CON; and b) PA and PA + WPC increased p-mTOR (Ser2481) levels, as well as p-rps6 (Ser235/236) relative to CON. Furthermore, our in vivo data demonstrates that PA tended to increase MPS and PA + WPC significantly increased MPS levels relative to CON. However, it is interesting that, while WPC increased MPS the greatest, PA+ WPC did not synergistically increase MPS levels in spite of the fact that p-p70s6k (Thr389) and p-rps6 (Ser235/236) were numerically the greatest in the combined fed group relative to PA and WPC alone. In this regard, we speculate that one of two phenomena are occurring: a) combined PA and WPC may alter the mTOR pathway activation dynamics relative to WPCinduced mTOR activation and, thus, peak MPS levels are shifted to the right or left of the $3 \mathrm{~h}$ post-feeding sampling point taken in the current study; or b) PA may actually interfere with WPC-induced increases in MPS. With regards to the latter point, PA and PA+ WPC rats presented decreased levels of $\mathrm{p}-4 \mathrm{EBP}-1$ relative to $\mathrm{CON}$; this being a potential mechanism whereby PA may have 'interfered' with WPC-induced increases in MPS. This finding is difficult to reconcile as in vitro investigations have demonstrated that PA treatment [14] or inhibition of PA production [5] results in the increased and decreased phosphorylation of 4EBP-1, respectively. Thus, it will be of future interest to examine how PA and PA + WPC affect mTORC1 signaling, namely 4EBP-1 phosphorylation, and MPS levels over multiple post-feeding time points in vivo.

PA, with or without WPC, differentially affect post-feeding skeletal muscle mRNAs related to anabolism and metabolism

Of the mRNA alterations that occurred with PA and/or PA + WPC, our major findings include: a) PA led to a robust post-feeding increase in p21Cip1 mRNA expression relative to $\mathrm{CON}$; b) PA and PA + WPC led to a significant increase in Glut-4 mRNA expression relative to $\mathrm{CON}$; and c) PA + WPC led to a significant increase in MuRF-1 mRNA expression relative to CON.

p21Cip1 gene expression is thought to promote satellite cell differentiation $[23,24]$, though others have 
shown that p21Cip1 gene expression is linked to protein synthesis and hypertrophy in post-mitotic kidney epithelial cells [25]. Furthermore, we [26] and others [27] have shown a $1-6 \mathrm{~h}$ robust increase in p21Cip1 mRNA expression after an acute bout of resistance exercise which suggests that this gene is being expressed from post-mitotic muscle fibers rather than differentiating satellite cells. Hence, it remains plausible that mTORC1 pathway activation may be linked to the mRNA expression of p21Cip1 which, in turn, may be related to enhanced translational mechanisms; this being a hypothesis which we have posited previously [28]. In support of this hypothesis, Nader et al. [29] have shown that mTOR increases the expression of cell cycle-related genes in post-mitotic myotubes in order to enhance ribosomal biogenesis. Therefore, it will be of further interest to examine if chronic PA supplementation leads to an increase in intramuscular markers of ribosome biogenesis, whether this effect is mitigated through an enhanced p21Cip1 gene expression, and whether this potential effect is causally-related to muscle hypertrophy evident with longer-term supplementation.

Our finding that PA + WPC ingestion increased MuRF-1 mRNA expression relative to $\mathrm{CON}$ is intriguing. While amino acids are anti-catabolic [30], other literature has shown that protein ingestion increases the mRNA expression of MuRF-1 mRNA in human skeletal muscle after resistance exercise [31,32]. Conversely, skeletal muscle MuRF-1 mRNA as well as markers of muscle protein synthesis has been reported to be lower in spinalcord-injured patients [33]; a finding which the authors suggest is linked to the tight regulation of muscle protein breakdown and synthesis rates. Thus, the results in the current study suggesting that PA + WPC ingestion increases the mRNA expression of MuRF-1 may represent a stimulation of greater muscle protein turnover (i.e., increased synthesis paralleled with increased breakdown rates) rather than an increase in atrophic mechanisms.

Finally, our finding that PA and PA + WPC modestly increased post-feeding Glut-4 mRNA is intriguing given the potential that this may be a manner whereby PA supplementation could potentially increase glucose sensitivity in an mTOR-dependent manner. To this end, insulin-like growth factor-1, a known activator of the mTORC1 signaling cascade, has been shown to increase Glut-4 mRNA in vitro [34]. Recent evidence also suggests that six weeks of cycling increases type II muscle fiber Glut-4 protein levels which were co-incident with increases in p-mTOR levels [35]. Thus, there seems to be interplay between mTORC1 activation and Glut-4 gene expression. To this end, it will be of further interest to examine if PA supplementation is capable of affecting serum glucose levels in rodent models or human populations with impaired glucose tolerance.

\section{Conclusions}

In summary, this is the first study to demonstrate that PA, with or without WPC co-ingestion, increases mTORC1 pathway activation. Furthermore, these data demonstrate that PA ingestion tends to increase MPS levels in vivo relative to the fasted condition. While these findings are novel, this study is not without its limitations including: a) the limited post-feeding time point interrogation, b) the lack of intramuscular PA data, and c) the absence of other PA and/or WPC dosages as well as the lack of an exercise stimulus. With regards to the limitation 'b', we attempted to use a commercial fluorometric assay to measure muscle PA levels, but we did not have confidence in the data due to the high background fluorometric signal that the assay buffer possessed. With regard to the last limitation, future studies are needed in order to examine if: a) pre-loading with PA and/or higher doses of PA further promotes skeletal muscle anabolism, and b) if higher doses of PA in an acute exercise setting facilitates greater increases in MPS relative to the dose used herein. To this end, mechanistic rat studies in our lab using a hind limb stimulation model of resistance exercise has proven to be effective for increasing MPS in the fasted state (unpublished observations) and, thus, this model could be a valuable tool that continues to explore the efficacy of PA in enhancing postexercise skeletal muscle anabolism. Given that mTORC1 pathway activation is integral in myoblast proliferation [36] and differentiation [37], examining how PA supplementation with resistance training affects myonuclear domain size, myonuclear number and satellite cell number is also warranted. It should also be noted that serum was not obtained from these animals and, given that Glut-4 mRNA transiently increases in PA-fed rats, future research should examine how dietary PA effects the postprandial hormonal milieu (i.e., potential PA-mediated increases in insulin). Finally, the potential metabolic effects of PA supplementation beyond skeletal muscle hypertrophy (i.e., the effects of PA supplementation on glucose tolerance and/or appetite given that hypothalamic mTORC1 activation is anorectic [38]) may unveil $\mathrm{PA}$ as a nutraceutical candidate in populations beyond resistance-trained athletes.

\section{Abbreviations \\ 4EBP-1/2: Eukaryotic initiation factor 4E-binding proteins 1/2; B2M: $\beta 2$ microglobulin; CON: Control; FRB domain: FKBP12-rapamycin binding; Glut-4: Glucose transporter-4; LBM: Lean body mass; MPS: Muscle protein synthesis; mRNA: messenger RNA; Mstn: Myostatin; mTOR: Mammalian target of rapamycin; MuRF-1: Muscle RING finger protein-1; p70s6k: p70s6 kinase; PA: Phosphatidic acid; Pgc-1a: Peroxisome proliferator-activated receptor gamma coactivator 1-alpha; rps6: ribosomal protein s6; RIPA buffer: Radioimmunoprecipitation assay; WPC: Whey protein concentrate.}




\section{Authors' contributions}

MDR outlined the experiments, provided funding from laboratory startup funds, assisted with in vivo experiments and helped draft the manuscript. $\mathrm{TAH}$ and RMM performed in vitro experiments and helped draft the manuscript. CBM, CDF, JCH, BSF, RPL, CML, JRS, ANK, and JMW assisted with in vivo experiments, tissue analysis, statistical analysis, data interpretation, and/or helped draft the manuscript. All authors read and approved the final manuscript.

\section{Acknowledgements}

The authors would like to thank Scott Hagerman and Chase Hagerman from Chemi Nutra, Inc. and Dr. Ralf Jäger and Dr. Martin Purpura from Increnovo LLC for their collaborative efforts. The authors would also like to thank Wes Kephart and Vincent Santucci for their assistance on gastrocnemius PA assays.

\section{Funding}

This project was supported by the laboratory start-up funds of M.D.R (in vivo data) as well as NIH grant (AR057347) to T.A.H. (in vitro data). Chemi Nutra provided additional funds for reagents involved with the in vitro arm of this study and paid for manuscript publication.

\section{Author details}

${ }^{1}$ School of Kinesiology, Auburn University, Auburn, AL, USA. ${ }^{2}$ Department of Comparative Biosciences, University of Wisconsin-Madison, Madison, WI, USA. ${ }^{3}$ Department of Health Sciences and Human Performance, University of Tampa, Tampa, FL, USA. ${ }^{4} 4$ LIFE Research, LLC, Sandy, UT, USA. ${ }^{5}$ Human Performance Laboratory, University of Central Florida, Orlando, FL, USA.

\section{Received: 3 April 2015 Accepted: 9 August 2015}

\section{Published online: 16 August 2015}

\section{References}

1. Rasmussen BB, Phillips SM. Contractile and nutritional regulation of human muscle growth. Exerc Sport Sci Rev. 2003;31(3):127-31.

2. Dibble CC, Manning BD. Signal integration by mTORC1 coordinates nutrient input with biosynthetic output. Nat Cell Biol. 2013:15(6):555-64. doi:10.1038/ncb2763.

3. Nishioka T, Frohman MA, Matsuda M, Kiyokawa E. Heterogeneity of phosphatidic acid levels and distribution at the plasma membrane in living cells as visualized by a Foster resonance energy transfer (FRET) biosensor. J Biol Chem. 2010;285(46):35979-87. doi:10.1074/jbc.M110.153007.

4. Wang X, Devaiah SP, Zhang W, Welti R. Signaling functions of phosphatidic acid. Prog Lipid Res. 2006;45(3):250-78. doi:10.1016/j.plipres.2006.01.005.

5. Fang $Y$, Vilella-Bach M, Bachmann R, Flanigan A, Chen J. Phosphatidic acid-mediated mitogenic activation of mTOR signaling. Science. 2001;294(5548):1942-5. doi:10.1126/science.1066015.

6. Hornberger TA, Chu WK, Mak YW, Hsiung JW, Huang SA, Chien S. The role of phospholipase $D$ and phosphatidic acid in the mechanical activation of mTOR signaling in skeletal muscle. Proc Natl Acad Sci U S A. 2006:103(12):4741-6. doi:10.1073/pnas.0600678103.

7. O'Neil TK, Duffy LR, Frey JW, Hornberger TA. The role of phosphoinositide 3-kinase and phosphatidic acid in the regulation of mammalian target of rapamycin following eccentric contractions. J Physiol. 2009:587(Pt 14):3691-701. doi:10.1113/jphysiol.2009.173609.

8. You JS, Lincoln HC, Kim CR, Frey JW, Goodman CA, Zhong XP, et al. The role of diacylglycerol kinase zeta and phosphatidic acid in the mechanical activation of mammalian target of rapamycin (mTOR) signaling and skeletal muscle hypertrophy. J Biol Chem. 2014;289(3):1551-63. doi:10.1074/ jbc.M113.531392.

9. Winter JN, Fox TE, Kester M, Jefferson LS, Kimball SR. Phosphatidic acid mediates activation of mTORC1 through the ERK signaling pathway. Am J Physiol Cell Physiol. 2010;299(2):C335-44. doi:10.1152/ajpcell.00039.2010.

10. Lehman N, Ledford B, Di Fulvio M, Frondorf K, McPhail LC, GomezCambronero J. Phospholipase D2-derived phosphatidic acid binds to and activates ribosomal p70 56 kinase independently of mTOR. FASEB J. 2007;21(4):1075-87. doi:10.1096/fj.06-6652com.

11. Hoffman JR, Stout JR, Williams DR, Wells AJ, Fragala MS, Mangine GT, et al. Efficacy of phosphatidic acid ingestion on lean body mass, muscle thickness and strength gains in resistance-trained men. J Int Soc Sports Nutr. 2012;9(1):47. doi:10.1186/1550-2783-9-47.
12. Joy JM, Gundermann DM, Lowery RP, Jager R, McCleary SA, Purpura M, et al. Phosphatidic acid enhances mTOR signaling and resistance exercise induced hypertrophy. Nutr Metab. 2014;11:29. doi:10.1186/1743-7075-11-29.

13. Shaw RJ. mTOR signaling: RAG GTPases transmit the amino acid signal. Trends Biochem Sci. 2008;33(12):565-8. doi:10.1016/j.tibs.2008.09.005.

14. Yoon MS, Sun $Y$, Arauz E, Jiang $Y$, Chen J. Phosphatidic acid activates mammalian target of rapamycin complex 1 (mTORC1) kinase by displacing FK506 binding protein 38 (FKBP38) and exerting an allosteric effect. J Biol Chem. 2011;286(34):29568-74. doi:10.1074/jbc.M111.262816.

15. Reagan-Shaw S, Nihal M, Ahmad N. Dose translation from animal to human studies revisited. FASEB J. 2008;22(3):659-61. doi:10.1096/fj.07-9574LSF.

16. Mobley CB, Fox CD, Ferguson BS, Pascoe CA, Healy JC, MCAdam JS, et al. Effects of protein type and composition on postprandial markers of skeletal muscle anabolism, adipose tissue lipolysis, and hypothalamic gene expression. J Int Soc Sports Nutr. 2015;12:14. doi:10.1186/s12970-015-0076-9.

17. Goodman CA, Mabrey DM, Frey JW, Miu MH, Schmidt EK, Pierre P, et al. Novel insights into the regulation of skeletal muscle protein synthesis as revealed by a new nonradioactive in vivo technique. FASEB J. 2011;25(3):1028-39. doi:10.1096/fj.10-168799.

18. Olszewski U, Deally A, Tacke M, Hamilton G. Alterations of phosphoproteins in $\mathrm{NCl}-\mathrm{H} 526$ small cell lung cancer cells involved in cytotoxicity of cisplatin and titanocene Y. Neoplasia. 2012;14(9):813-22.

19. Victoni $T$, Gleonnec $F$, Lanzetti $M$, Tenor $H$, Valenca $S$, Porto LC, et al, Roflumilast $\mathrm{N}$-oxide prevents cytokine secretion induced by cigarette smoke combined with LPS through JAK/STAT and ERK1/2 inhibition in airway epithelial cells. PLoS One. 2014;9(1), e85243. doi:10.1371/journal.pone.0085243.

20. McGinnis GR, Ballmann C, Peters B, Nanayakkara G, Roberts M, Amin R, et al. Interleukin-6 mediates exercise preconditioning against myocardial ischemia reperfusion injury. Am J Physiol Heart Circ Physiol. 2015;308(11):H1423-33. doi:10.1152/ajpheart.00850.2014.

21. Soliman GA, Acosta-Jaquez HA, Dunlop EA, Ekim B, Maj NE, Tee AR, et al mTOR Ser-2481 autophosphorylation monitors mTORC-specific catalytic activity and clarifies rapamycin mechanism of action. J Biol Chem. 2010;285(11):7866-79. doi:10.1074/jbc.M109.096222.

22. Mobley CB, Fox C, Pascoe C, Ferguson B, Healy J, Lockwood C, et al. Differential effects of whey protein concentrate and hydrolyzed whey/egg protein blends on post-prandial markers of insulin signaling and skeletal muscle anabolism in rats. FASEB J. 2014;28(1):LB439.

23. Hawke TJ, Jiang N, Garry DJ. Absence of p21CIP rescues myogenic progenitor cell proliferative and regenerative capacity in Foxk1 null mice. J Biol Chem. 2003;278(6):4015-20. doi:10.1074/jbc.M209200200.

24. Hawke TJ, Meeson AP, Jiang N, Graham S, Hutcheson K, DiMaio JM, et al. p21 is essential for normal myogenic progenitor cell function in regenerating skeletal muscle. Am J Physiol Cell Physiol. 2003;285(5):C1019-27. doi:10.1152/ajpcell.00055.2003.

25. Fan YP, Weiss RH. Exogenous attenuation of p21(Waf1/Cip1) decreases mesangial cell hypertrophy as a result of hyperglycemia and IGF-1. J Am Soc Nephrol. 2004;15(3):575-84

26. Roberts MD, Dalbo VJ, Hassell SE, Brown R, Kerksick CM. Effects of preexercise feeding on markers of satellite cell activation. Med Sci Sports Exerc. 2010;42(10):1861-9. doi:10.1249/MSS.0b013e3181da8a29.

27. Hulmi JJ, Kovanen V, Selanne H, Kraemer WJ, Hakkinen K, Mero AA. Acute and long-term effects of resistance exercise with or without protein ingestion on muscle hypertrophy and gene expression. Amino Acids. 2009:37(2):297-308. doi:10.1007/s00726-008-0150-6

28. Roberts MD, Dalbo VJ, Kerksick CM. Postexercise myogenic gene expression: are human findings lost during translation? Exerc Sport Sci Rev. 2011:39(4):206-11. doi:10.1097/JES.0b013e31822dad1f.

29. Nader GA, McLoughlin TJ, Esser KA. mTOR function in skeletal muscle hypertrophy: increased ribosomal RNA via cell cycle regulators. Am J Physiol Cell Physiol. 2005;289(6):C1457-65. doi:10.1152/ajpcell.00165.2005.

30. Herningtyas EH, Okimura $Y$, Handayaningsih AE, Yamamoto D, Maki T, lida K, et al. Branched-chain amino acids and arginine suppress MaFbx/atrogin-1 mRNA expression via mTOR pathway in $\mathrm{C} 2 \mathrm{C} 12$ cell line. Biochim Biophys Acta. 2008;1780(10):1115-20. doi:10.1016/j.bbagen.2008.06.004.

31. Areta JL, Burke LM, Ross ML, Camera DM, West DW, Broad EM, et al. Timing and distribution of protein ingestion during prolonged recovery from resistance exercise alters myofibrillar protein synthesis. J Physiol. 2013;591(Pt 9):2319-31. doi:10.1113/jphysiol.2012.244897.

32. Dalbo VJ, Roberts MD, Hassell S, Kerksick CM. Effects of pre-exercise feeding on serum hormone concentrations and biomarkers of myostatin and 
ubiquitin proteasome pathway activity. Eur J Nutr. 2013;52(2):477-87. doi:10.1007/s00394-012-0349-x.

33. Leger B, Senese R, Al-Khodairy AW, Deriaz O, Gobelet C, Giacobino JP, et al Atrogin-1, MuRF1, and FoXO, as well as phosphorylated GSK-3beta and 4E-BP1 are reduced in skeletal muscle of chronic spinal cord-injured patients. Muscle Nerve. 2009;40(1):69-78. doi:10.1002/mus.21293.

34. Valverde AM, Navarro P, Teruel T, Conejo R, Benito M, Lorenzo M. Insulin and insulin-like growth factor I up-regulate GLUT4 gene expression in fetal brown adipocytes, in a phosphoinositide 3-kinase-dependent manner. Biochem J. 1999;337(Pt 3):397-405.

35. Stuart CA, Howell ME, Baker JD, Dykes RJ, Duffourc MM, Ramsey MW, et al. Cycle training increased GLUT4 and activation of mammalian target of rapamycin in fast twitch muscle fibers. Med Sci Sports Exerc. 2010;42(1):96-106. doi:10.1249/MSS.0b013e3181ad7f36.

36. Rodgers JT, King KY, Brett JO, Cromie MJ, Charville GW, Maguire KK, et al mTORC1 controls the adaptive transition of quiescent stem cells from $\mathrm{G} 0$ to G(Alert). Nature. 2014;509(7505):393-6. doi:10.1038/nature13255.

37. Miyabara EH, Conte TC, Silva MT, Baptista IL, Bueno Jr C, Fiamoncini J, et al Mammalian target of rapamycin complex 1 is involved in differentiation of regenerating myofibers in vivo. Muscle Nerve. 2010;42(5):778-87. doi:10.1002/mus.21754.

38. Watterson KR, Bestow D, Gallagher J, Hamilton DL, Ashford FB, Meakin PJ, et al. Anorexigenic and orexigenic hormone modulation of mammalian target of rapamycin complex 1 activity and the regulation of hypothalamic agoutirelated protein mRNA expression. Neurosignals. 2013;21(1-2):28-41. doi:10.1159/000334144

\section{Submit your next manuscript to BioMed Central and take full advantage of:}

- Convenient online submission

- Thorough peer review

- No space constraints or color figure charges

- Immediate publication on acceptance

- Inclusion in PubMed, CAS, Scopus and Google Scholar

- Research which is freely available for redistribution 